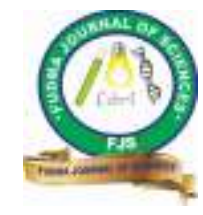

FUDMA Journal of Sciences (FJS)

ISSN online: $2616-1370$

ISSN print: 2645 - 2944

Vol. 4 No. 2, June, 2020, pp 490 - 498

DOI: https://doi.org/10.33003/fjs-2020-0402-231

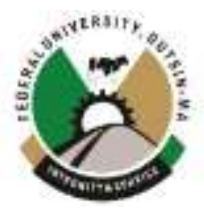

\title{
DFT COMPUTATION OF THE BAND STRUCTURE AND DENSITY OF STATE FOR ZnO HALITE STRUCTURE USING FHI-aims CODE.
}

\author{
${ }^{* 1}$ Adamu, M. A., ${ }^{1}$ Lawal, K., and ${ }^{2}$ Saminu, A. \\ ${ }^{1}$ Department of Physics, Federal University Dutsin-Ma, Katsina, Nigeria. \\ ${ }^{2}$ School of Basic and Remedial Studies, Federal Polytechnic Kaura Namoda, Zamfara State. \\ ${ }^{*}$ Corresponding Author’s Email: adamuaminu532@gmail.com, +2348137006474
}

\begin{abstract}
This research work is on Density Functional Theory (DFT) within Local Density Approximation as parameterised by Perdew and Wang (pw-lda). The calculation was performed using Fritz Haber Institute Abinitio Molecular Simulations (FHI-aims) code based on numerical atomic-centered orbital basis sets. The electronic band structure, density of state (DOS) and band gap energy were calculated for $\mathrm{ZnO}$ compound. The band structure and Density of States (DOS) diagrams are plotted from the calculated equilibrium lattice parameters. The experimentally lattice constant values were used to calculate the minimum total energy. The calculated electronic band structure results show that $\mathrm{ZnO}$ (Halite) is an indirect semiconductor with energy band gap of $0.89 \mathrm{eV}$. Hence, the HOMO is $-0.863382 \mathrm{eV}$ at L_symmetry point and LUMO is $0.0239417 \mathrm{eV}$ at $\Gamma$ - point. The DOS energy level within the compound shows considerable high state of electron occupation and the DOS observed around the Fermi level at zero level indicate that it has conducting properties. In general, FHI-aims code has shown better accuracy and prediction of band structure calculation within reasonable computational methods.
\end{abstract}

Keywords: Band structure, Density of state, DFT, ZnO and Halite.

\section{INTRODUCTION}

Semiconductor materials are available for different purposes. Germanium gets popular due to possession of properties such as low melting point and lack of natural occurring germanium oxide to avoid the surface from electrical leakage whereas silicon dominates the commercial market for its better fabrication technology and application to integrated circuits for different reasons. But the rapid growing world needs speed along with technology. The world now demands a material that should possess fundamental properties such as larger band gap, higher electron mobility as well as higher breakdown field strength for technological industrialization. Zinc Oxide $(\mathrm{ZnO})$ fulfills the required properties. The understanding of the physical properties of interacting many body systems is one of the most important achievements of physics after the establishment of quantum mechanics in the mid 1920's. One of the most immediate consequences of the periodic structure of crystalline solids is the arrangement of the electronic states within bands. This band has a specific importance for semiconductors as many properties of semiconductors are determined by only a small number of these bands. Band structure as a concept in solid state physics assumed that for bands to be continuous a large piece of material must be considered, therefore it is extended to system along reduced dimension such as two or three-dimension electron systems. Another assumption involves the homogeneity of a system in which the notion of a band structure as an intrinsic property of a material is homogeneous in some ways; this means that band structure describes the bulk system inside a uniform piece of material. It also assumes non interactivity of a system which describes single electron states that travel in a static potential without dynamically interacting with lattice vibrations, electrons, photons, etc. Minden H. T. (1970). The excitation in a crystalline solid can be characterized by a Bloch vector usually referred to as structure of the electronic energy levels (band structure). This electronic structure determines the chemical bonds which keep the atoms together. To study the ground state and other related properties of electronic systems, a quantum model is needed for these systems, Density Functional Theory (DFT) is such a model. It involves using the probability density functions of the electrons in a molecule to determine other properties of the molecules. Instead of solving problems with wave equations for each electron it uses the probabilities of electrons being at different locations. DFT works by minimizing functions of functions to determine the ground state of a molecule. Instead of using functional based upon each individual electron, a pseudo potential or basis set is used to simplify the calculation. DFT is in principle exact for the ground-state energy and the electron density, although in practice approximations become necessary. Hence, DFT contributed enormously to the development of condensedmatter physics and quantum chemistry. 
Therefore, DFT has become the workhorse in electronic band structure calculations and FHI-aims computer code has been used in this work to address this many particle problem, with more efficiency and accuracy by implementing all-electron density based on numeric atomic-centered orbital basis sets which allow the actual predictions for real materials using a computer program code. The code answers many possible general purpose electronic structure problems by varying the treatment of exchange correlation in Local density Approximation (LDA) and Generalized Gradient Approximation (GGA). This invokes the LDA as exchange correlation functional given by slater and the correlation energy of the electron given by Perdew and wang (Perdew and Wang 1992). Its success rate has helped in producing accurate results with reasonable computational effort.

\section{Theoretical Frame Work}

Traditional methods in electronic structure theory, in particular Hatree-Fock theory and its descendants are based on the complicated many-electron wave function. The main objective of DFT is to replace the many-body electronic wave function with the electronic density as the basic quantity. In 1965, Walter Kohn and Lu Sham proposed an educated guess that later yielded results in which they reformulated the problem in a more familiar form and opened the way to practical application of DFT. The system of interacting electrons is mapped onto a fictitious system of non-interacting electrons having the same ground state charge density $n(r)$. For a system of noninteracting electrons the ground-state charge density is represented as a sum over one-electron orbital's (the KS orbital's) $\psi_{i}$, (Giannozzi, 2005).

$$
n(r)=2 \sum_{i}\left|\psi_{i}(r)\right|^{2}
$$

Where $i$ runs from 1 to N/2. If we assume double occupancy of all states, and the Kohn-Sham orbitals are the solution to the Schrödinger equation:

$$
\left(-\frac{\hbar^{2}}{2 m} \nabla^{2}+V_{K S}(r)\right) \psi_{i}(r)=\in_{i} \psi_{i}(r)
$$

$\mathrm{m}$ is the electron mass.

The existence of a unique potential $V_{K S}$ in equation (2) having $n(r)$ as its ground state charge density is a consequence of the $H$ - $K$ theorem, which holds irrespective of the form of electron-electron interaction $U$.

In closed systems, suppose there is an even number of electrons, so that they all can be paired up and the external potential V(r) is independent of spin. Spin-up and spin-down contribute equally to the total density:

$$
n(\uparrow)(r)=n(\downarrow) \frac{1}{2}(n)
$$

the density can also be written as:

$$
n(r)=\sum_{i=1}^{N / 2} / \Psi_{i}(r) P^{2}
$$

and the kinetic energy as

$$
T_{S}=-\frac{1}{2} \sum_{i=1}^{N / 2}<\Psi_{i} /(r) / \nabla^{2} / \Psi_{i}(r)>
$$

The problem is now to determine $V K S(r)$ for a given $n(r)$. To solve this problem it is convenient to rewrite the energy functionals as:

$$
\mathrm{E}=\mathrm{T}[\mathrm{n}(\mathrm{r})]+E_{H}[n(r)]+E_{x c}[n(r)]+\int n(r) V_{(r)} d_{r}
$$




\section{METHODOLOGY}

FHI-aims Code is used in the Calculations of this work. It is a computer program package for computational materials science based on quantum-mechanical first principles. The main production method is the use of DFT to compute the total energy and derived quantities of molecules and solids of condensed matter in its electronic ground state. The focus of this work is to use DFT to estimate band structure and DOS of halite $\mathrm{ZnO}$ using methods of the local density approximations (LDA) in the exchange-correlation potential as parameterized by Perdew and Wang LDA (pw-lda).

The full algorithmic framework embodied in the FHI-aims computer program package is described in (Blum et'al 2009). The algorithms are based on numerically tabulated atomcentered orbitals (NAOs) to capture a wide range of molecular and materials properties from quantum-mechanical first principles and all-electron/full-potential treatment that is both computationally efficient and accurate. This is achieved for periodic and cluster geometries on equal footing, including relaxation and ab initio molecular dynamics.

Next, we set basic parameters in control in for periodic calculations. Finally, we compare total energies of different $\mathrm{ZnO}$ bulk geometries. In setting up the geometry.in file of a periodic structure in FHI-aims, the lattice vectors as well as the atomic positions in the unit cell are specified. The electronic band structure of the stable phases of the semiconductor was then calculated along the high symmetry lines of the Brillouin zone by computing the control.in settings to calculate the bandstructures and density of state.

Physically $\mathrm{ZnO}$ exists in three different structural phases, at room temperature Wurtzite (B4) is stable, high pressure rock salt/halite (B1) and zinc-blende (B3) (Bakhtiar, Afaq, Ahmed and Naseem, 2012).

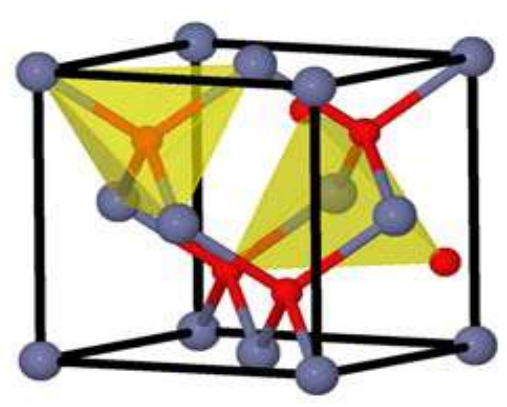

(a)Zinc-blend

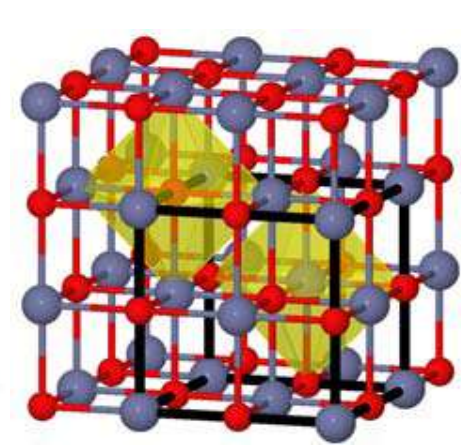

(b) Rock-salt

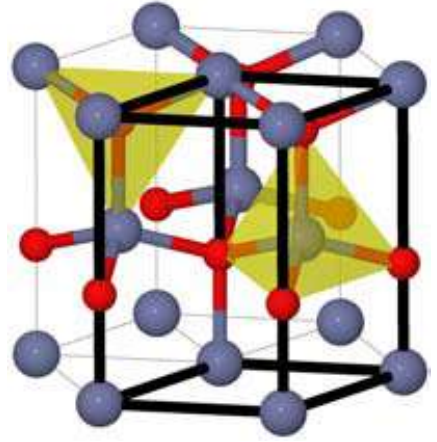

(c) Wurtzite

Fig 1: Crystalline structures of the three $\mathrm{ZnO}$ structures

The experimental lattice constant use for the computation for Halite, Wurtzite and Zinc-blend are: $a=4.2825 \AA$ (Koster et'al,

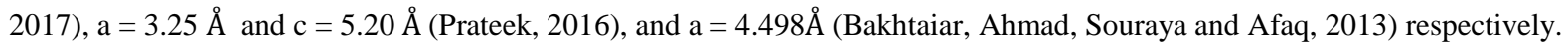

The calculation was performed by using Brillouin-zone of $12 \times 12 \times 12 \mathrm{k}$-grid of the SCF convergence. The total energy for each iteration step is then recorded in a table.

\section{RESULTS AND DISCUSSION}

The results obtained are based on the output files from the FHI-aims code used for computation. This is used to deduce the total energies against the number of iterations. Graphs were plotted to obtain the optimized parameters for the $\mathrm{ZnO}$ (halite, Wurtzite, and zinc blend) respectively. Ground state energies of $\mathrm{ZnO}$ (halite, Wurtzite and zinc blend) crystal structures are shown in Table 1 .

Table 1: Ground state energy of $\mathrm{ZnO}$ crystals structure

\begin{tabular}{|l|l|l|l|}
\hline Computation & Functional & Crystal structure & Ground state energy \\
\hline Light & Pw_lda & Halite & \\
\hline Light & Pw_lda & Wurtzite & -50836.95263588 \\
\hline Light & Pw_lda & Zinc blend & -50830.14290881 \\
\hline
\end{tabular}


From the table 1 we choose the most stable structure and then relaxed it for the computation of DOS and band structure based on the computation results, zinc blend is the most stable structure due to its highest ground state energy. However, its relaxation took approximately 19 to 20 hours but still the structure relaxation did not converge. Therefore, we decided to use the next stable structure (halite). The Halite structure relaxation was achieved successfully. We therefore compute only the DOS and band structure of $\mathrm{ZnO}$ (halite).

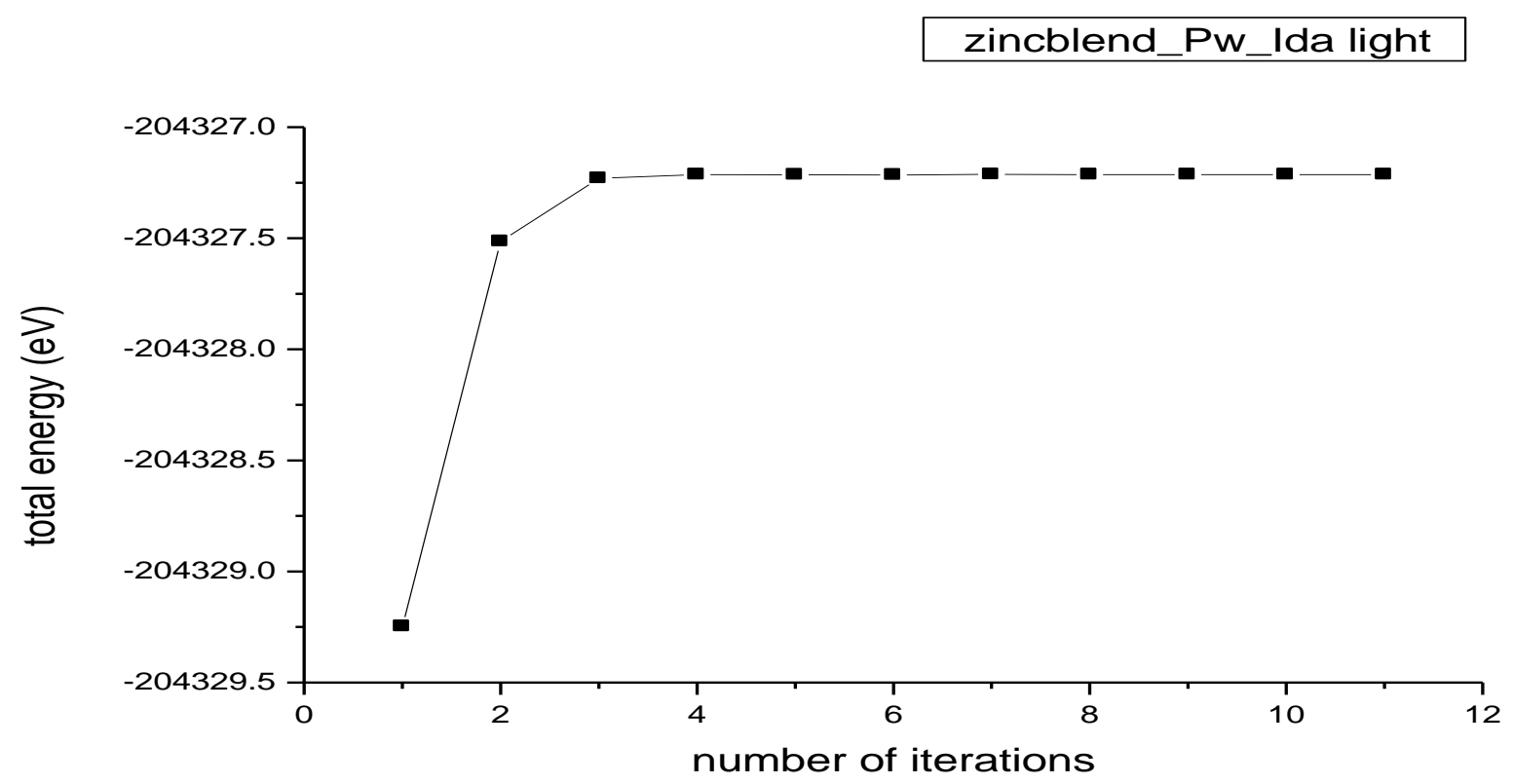

Figure 2: Binding curve of total energy against number of iterations for zinc blend.

Fig. 2 shows the binding curve of pw_lda for zinc blend. It was found that the total energy for zinc blend became stable at the 3rd iteration having twelve (12) converged iterations; however it also shows that the total energy increases as the number of iterations increases and converges with stability from 3rd iteration to the last iteration.

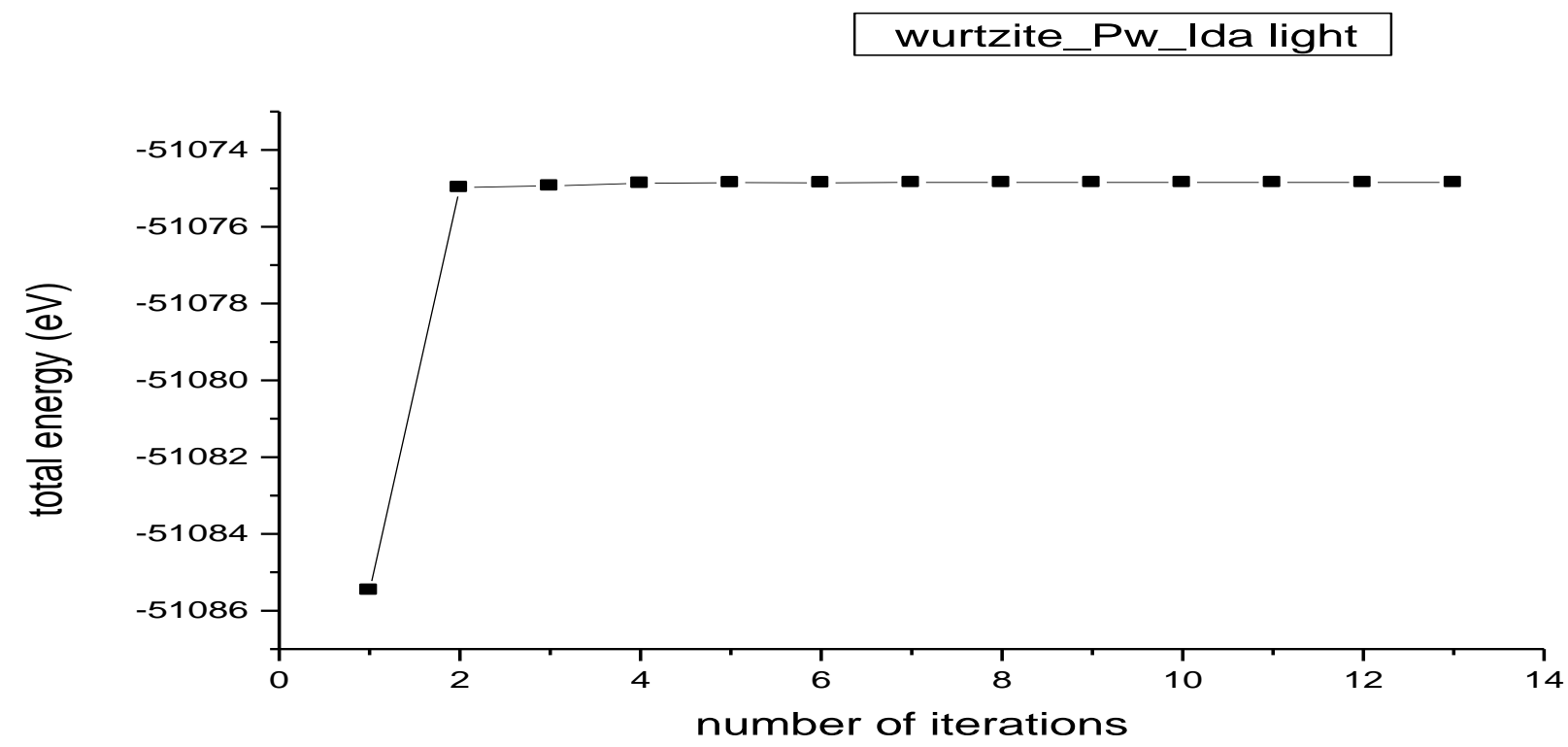

Figure 3: Binding curve of total energy against number of iterations for Wurtzite. 
Similarly, Fig. 3 shows the binding curve of pw_lda for Wurtzite. It was found that the total energy for Wurtzite became stable at the 2 nd iteration having fourteen (14) converged iterations. However, it also shows that the total energy increases as the number of iterations increases and converges with stability from 2 nd iteration to the last iteration.

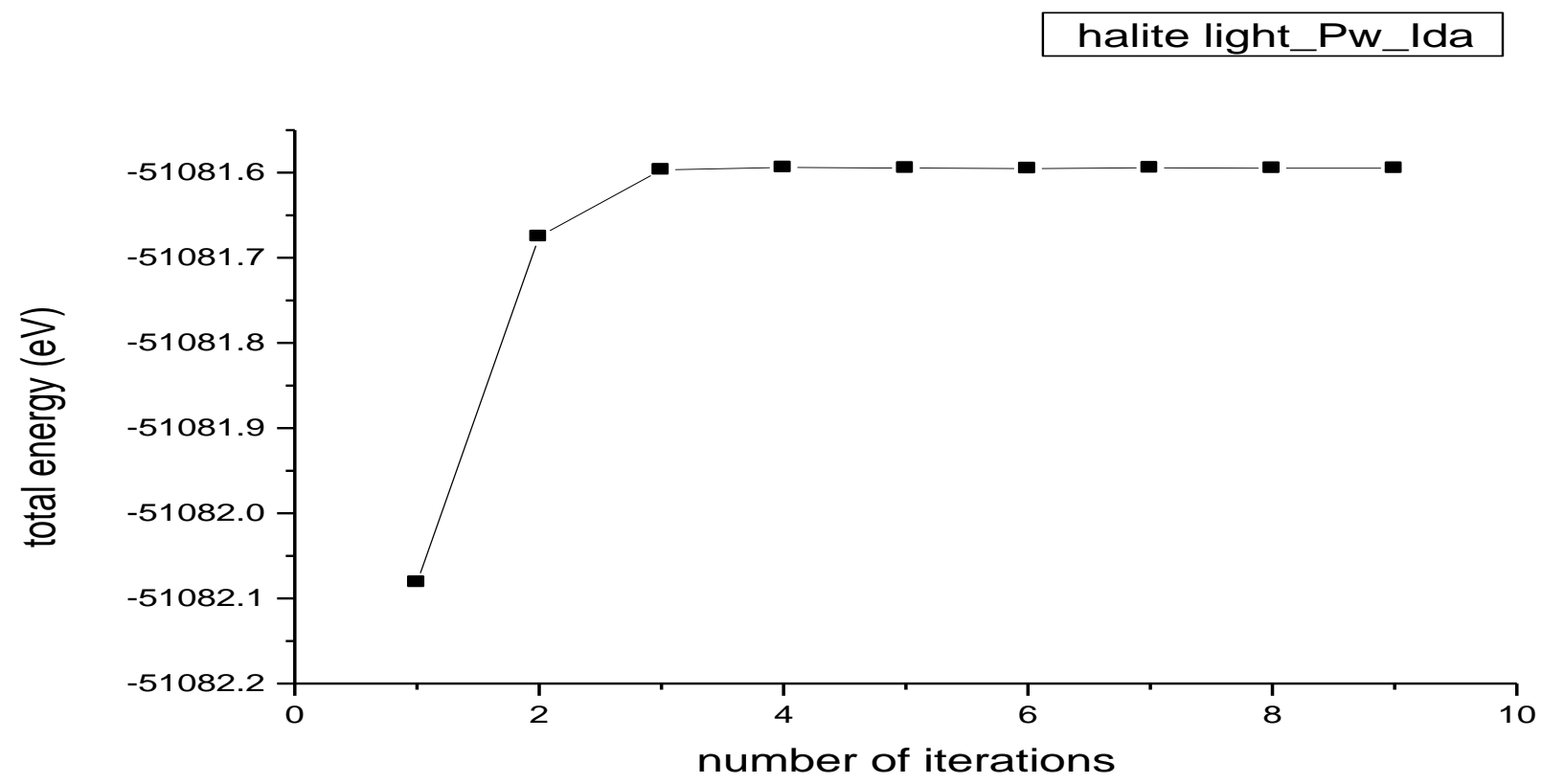

Figure 4: Binding curve of total energy against number of iterations for halite

Fig.4 shows the binding curve of pw_lda for halite. It was found that the total energy for halite became stable at the 3rd iteration having ten (10) converged iterations; however it also shows that the total energy increases as the number of iterations increases and converges faster with stability from the 3rd iterations.

FHI-aims aimsplot.py script was used to plot the band structure. The position of the Fermi level in the band structure of these crystals is shown by the zero on the energy scale and that of symmetry points are indicated by vertical lines on the band structure as shown in figure 5. There is an important characteristic of the band structure, namely the range of energies where there are no electronic states across the entire Brillouin zone; this is the band gap. We notice that the band below zero shows that all the bands are fully occupied. The Fermi level within the band gap shows that all state below it remain occupied and all state above remain unoccupied. This is the hallmark of metallic behaviors that is, the availability of state immediately below and immediately above the Fermi level, which makes it possible to excite electrons thermally. 

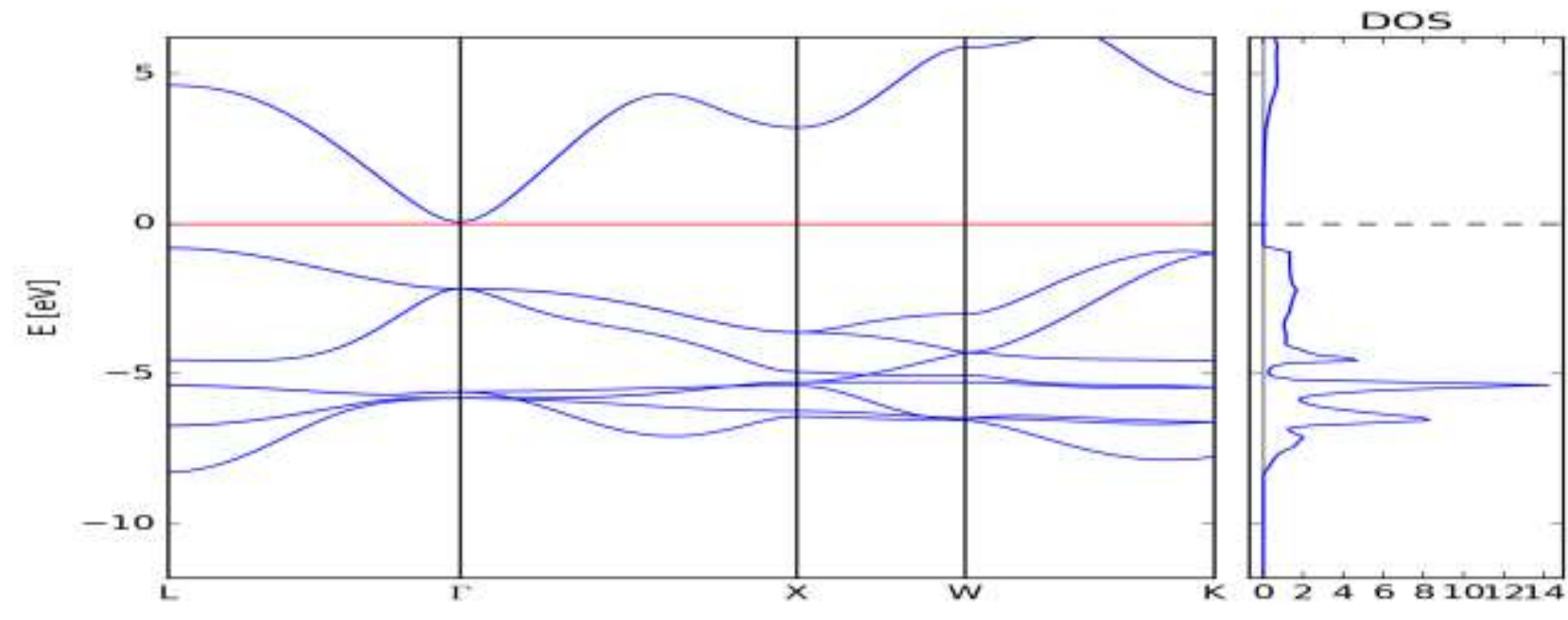

Figure 5: The Band structure and DOS of $\mathrm{ZnO}$ (Halite)

Figure 5 shows the $\mathrm{ZnO}$ band structure and DOS.

Table 2: the overall basic results of $\mathrm{ZnO}$

\begin{tabular}{|l|l|l|l|l|l|}
\hline Crystal structures & $\begin{array}{c}\text { HOMO }(\mathrm{VBM}) \\
(\mathrm{eV})\end{array}$ & $\begin{array}{l}\text { LUMO }(\mathrm{CBM}) \\
(\mathrm{eV})\end{array}$ & $\begin{array}{l}\text { HOMO-LUMO } \\
\text { gap }(\mathrm{eV})\end{array}$ & $\begin{array}{l}\text { Total Energy } \\
(\mathrm{eV})\end{array}$ & $\begin{array}{l}\text { Previous } \\
\text { researched Band } \\
\text { gap }(\mathrm{eV})\end{array}$ \\
\hline Halite & -7.01401502 & -6.14433689 & 0.86967813 & -50836.952636 & 0.850 \\
\hline Wurtzite & -6.40175066 & -6.39839579 & 0.00335487 & -50830.1429088 & 0.759 \\
\hline $\mathrm{Zi}$ & -6.87380697 & -6.15914032 & 0.71466665 & -203348.704978 & 0.628 \\
& & & & & \\
\end{tabular}

We also notice that the band gap in $\mathrm{ZnO}$ is narrow which shows that the semiconductor becomes more ionic. This effect arises from dehybridization accompanying the change in bonding from covalent to ionic. The smaller gap is important for transport properties in Halite semiconductor and has been observed to have subtle effect on the reflectivity spectrum of Halite (Choen and Chelikowsky 1988). The $\mathrm{ZnO}$ has two electrons in the $\mathrm{Zn}$ orbital and six electrons in the $\mathrm{O}$ orbital, that is, the highest occupied molecular orbital (HOMO) in the valance band is made up of $\mathrm{d}$ orbital while the conduction band or the lowest occupied molecular orbital (LUMO) is formed by only sp hybridized orbital. This again gives rise to a difference in the nature of the HOMO and LUMO which retards the return of the excited electron to the valence band however, makes the state near the valence band maximum have $\mathrm{p}$ bonding character which are associated with more electronegative element in the solid, while those near the conduction band minimum have $\mathrm{p}$ anti-bonding character and are associated with less electro negativity. The energy band gap is seen as the difference between the conduction band and the valence band which is calculated (estimated HOMO-LUMO $=0.0239417+0.863382$ $=0.8873237$ ) to be $0.89 \mathrm{eV}$. This research work agrees with other previous research work such as the band gap of halite $(\mathrm{ZnO})$ which is found to be $0.85 \mathrm{eV}$ (LDA) using WIEN2k tool by Muhammad and Afaq, 2015. And also $0.65 \mathrm{eV}$ (GGA) according to Muhammad and Afaq, 2015. However other values are found by other researchers such as $1.537 \mathrm{eV}$ (Bukhtiar, Ahmad, Souraya, Shaari and Afaq, 2013).

From figure: 5 above we notice that halite Fermi energy is $0.08500 \mathrm{eV}$ and the HOMO is at L_symmetry point while the LUMO is at $\Gamma$ - point. Since the valence band maximum and the conduction band are on different symmetry point it shows that halite is an indirect band gap, the effect of the narrow band width was observed to be stronger at the $\Gamma-$ point and it grows 
into $\mathrm{L}, \mathrm{W}$ and $\mathrm{K}$ symmetries without forming any hybridization. $\mathrm{ZnO}$ is ionic and due to its increasing ionic character, have more charges transfer. These also make it share metallic and covalent properties.

Figure.5: also shows the Density of state of $\mathrm{ZnO}$ as a halite structure semiconductor; we divide the density of state into three general regions while analyzing. The first region is the most tightly bound energy band where electron states corresponding to this band are strongly localized on the anion state. The next region of noticed is a peak arising from the onset of the second valence band which shows there is no energy variation along the symmetry direction; in fact, it is very flat over the entire square face of the Brillouin zone. The character of state associated with the second valence band changes from predominantly cation slike states at the bond edge to predominantly anion p-like state at the band maximum. The third region of interest in the density of state extends from the onset of the third valence band (at about $4 \mathrm{eV}$ below the valence band maximum) to the valence band maximum. This region encompasses the top two valence bands and is predominantly p-like and is associated with anion state.

The band gap of ZnO has narrow band gap and can't be use in high temperature applications. They act as a promising candidate in semiconductor technology due to its narrow and indirect band gap (Hellwege et, al. 1982). This also makes it an active material in the manufacture of optoelectronic devices and also in LEDs etc. It has also gained importance in the technology applications of short wave length range of higher power and high frequency electronic devices. In particular $\mathrm{ZnO}$ is a basic material for light emitting diodes, lasers in the blue and ultraviolet range of the spectrum, optical pumping structures, photo detectors and hetero structure).

\section{CONCLUSION}

This work has successfully employed Density Functional Theory method to calculate and estimate the band structure of $\mathrm{ZnO}$ (Halite) using FHI-aims which was successfully installed and the knowledge of the input parameters which include the geometry.in and the control.in was carefully optimized for the band structure studies. All calculations were carried out using FHI-aims code which works on Linux based operating system. In the calculation, Local Density Approximation (LDA) has been used to approximate the exchange correlation energy which varied the treatment of exchange correlation (LDA) to Kohn-Sham DFT leaving all other settings constant. The experimentally lattice constant parameter values was used to calculate the minimum total energy and tested for different $\mathrm{k}$ grids. The minimum total energy obtained from the experimental lattice constant of $\mathrm{ZnO}$ (halite) $2.14125 \AA$ results in $-50836.95263588 \mathrm{eV}, \mathrm{ZnO}$ (Wurtzite) $\mathrm{a}=3.25 \AA, \mathrm{c}=5.20 \AA$ results in $-50830.14290881 \mathrm{eV}$, and $\mathrm{ZnO}$ (zinc blend) $\mathrm{a}=4.498 \AA$ results in $-203348.70497768 \mathrm{eV}$. The result obtained shows that $12 * 12 * 12$ k-grids enable the energy stability of the semiconductors in diamond structure with less computational time. The calculated electronic band structure results show that $\mathrm{ZnO}$ halite is an indirect band gap semiconductor with $0.89 \mathrm{eV}$. The DOS energy level within the semiconductors shows considerable high state of electron occupation and the DOS observed around the Fermi level for the semiconductors at zero level indicate that they have conducting properties (Qin et al 2009).In general, FHI-aims code has predicted the band structure and DOS calculation within a reasonable computational time when compared to some other DFT theoretical programs observed in literature.

\section{REFERENCES}

Abdu S.G., Adamu M.A, and Onimisi M.Y.(2018). DFT Computations of the lattice constant, stable atomic structure and the ground state energy per atom of fullerenes (c60). Science World Journal Vol $13 \quad$ (No 1$) \quad 2018$. www.scienceworldjournal.org, ISSN 1597-6343

ABDU, S.G. (2010). Hartree-Fock Solutions of the Hydrogen, Helium, Lithium, Beryllium and Boron atoms. Nigerian Journal of Physics Vol. 21 (2) 2010.

Aungwa F, Ahoume B.A. and Danladi E. (2017). Cohesive Energy calculation of Gallium-Arsenide and AluminniumArsenide; DFT study. Journal of the Nigerian Association of Mathematical Physics, Volume 39. pp 305-312

Aungwa F, (2016). Density functional theory computation of the cohesive energies of $\mathrm{NaCl}, \mathrm{SiO} 2$ and $\mathrm{Al}$

Bakhtiar Ul Haq, R. Ahmed, Souraya Goumri-Said, A. Shaari \& A. Afaq (2013): Electronic structure engineering of $\mathrm{ZnO}$ with the modified Becke-Johnson exchange versus the classical correlation potential approaches, Phase Transitions: A Multinational Journal, DOI:10.1080/01411594.2012.755183

Bakhtiar UI. HAQ, AFAQ, A., AHMED, R., \& NASEEM, S. (2012). A Comprehensive DFT Study of Zinc Oxide in Different Phases. International Journal of Modern Physics C, 23(06), 1250043. DOI:10.1142/S012918311250043X

Blum, V., Gehrke R, Hanke F, Havu P, Havu V, Ren X, Reuter K, Scheffler M. (2009). "Ab initio molecular simulations with numeric atom-centered orbitals" Computer Physics Communications, 180, 2175-2196

Cohen, M.L., Chelikowsky, J. R. (1988). Electronic structure and Optical Properties of Semiconductors

Giannozzi, p. (2005). "Density functional theory for electronic structure calculations" Universit`a di Pisa, Italy. 
Hellwege, K. H, Madelung, O. and Landolt B. (1982). New series, Springer Group III. Vol 17a, Berlin.

Hohenberg, P. and Kohn, W. (1964) Inhomogeneous Electron Gas, Phys. Rev., vol.136, no. 3B, pp. B864-B871,

Kohn, W. and Sham, L.J. (1965) "Self-Consistent Equations Including Exchange and Correlation Effects," Phys. Rev., vol. 140, no. 4A, pp. A1133-A1138,

Minden, H. T. (1970). Some Optical Properties of Aluminum Arsenide. Applied Physics Letters. Vol. 17, Issue 9. pg. 358360.
Muhammad A. and Afaq A. (2015). First Principles Band-gap Calculations of $3 \mathrm{~d}$ Transition Metals-added $\mathrm{ZnO}$ Materialstoday: Proceedings Volume 2, Issue 10, Part B, 2015, Pages 5128-5131, https://doi.org/10.1016/j.matpr.2015.11.009.

Perdew, J.P., Wang, Y. (1992) Accurate and simple analytic representation of the electron-gas correlation energy. Phys. Rev. B 45(23), 13244-13249.

Prateek U. and Kirti V. (2016), India International Journal of Emerging Technology in Computer Science \& Electronics (IJETCSE) ISSN: 0976-1353 Volume 21. 


\section{APPENDIX}

The structures lattice vectors and atomic coordinates used are shown in Table 3.

Table 3: Structure Information

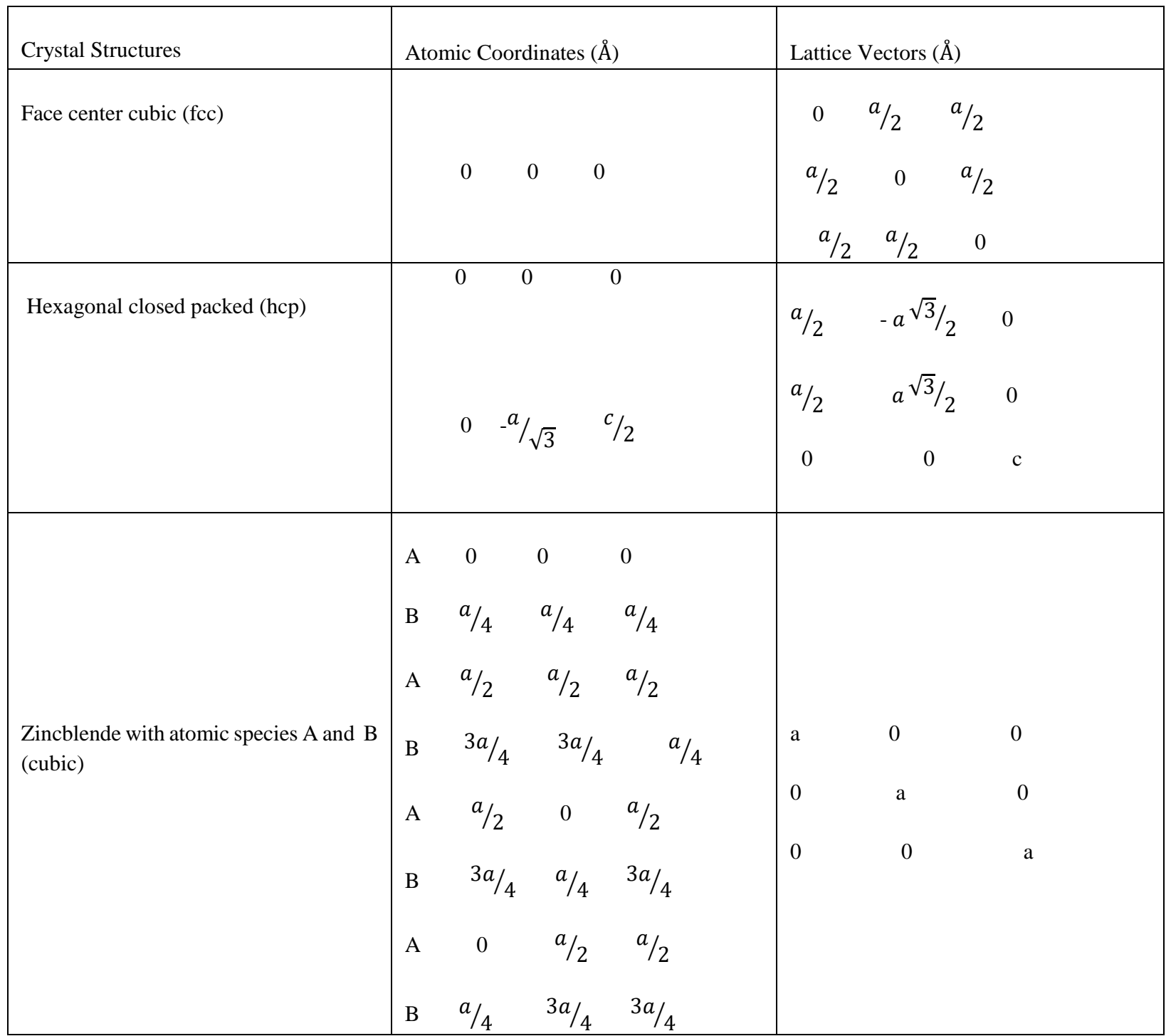
use, distribution, and reproduction in any medium, provided the original work is cited appropriately. 